\title{
Resección duodenal distal: Análisis de nuestra experiencia con una cirugía infrecuente
}

\author{
Gabriela S. Ochoa S. ${ }^{1}$, Fernando Crovari E. ${ }^{1}$ y Eduardo Briceño V. ${ }^{1}$
}

'Departamento de Cirugía Digestiva, Pontificia Universidad Católica de Chile. Santiago, Chile.

Recibido 2020-06-10 y aceptado 2020-09-21

Correspondencia a:

Dr. Eduardo Briceño V eduardo.ebriceno@gmail.com

\section{Distal duodenal resection: Analysis of our experience with infrequent surgery}

Introduction: Distal duodenal resection is a complex and infrequent surgical technique used for the treatment of duodenal or extraduodenal pathologies that do not compromise the greater duodenal papilla, the most frequent indication being the neoplastic cause. Aim: To publicize our experience with this surgical technique. Materials and Method: Series of patients undergoing a distal duodenal resection between 2009-2020 at the UC Clinical Hospital. Results: Total 12 patients, median age 56 years. Preoperative diagnosis: primary duodenal tumors (4), tumors with duodenal involvement due to contiguity (5), and emergency pathologies (3). The approach was open in 9 patients and laparoscopic in 3 (1 converted). D3-D4 was resected in 10 patients, 1 included part of D2 and 2 only D4. The duodenojejunal anastomosis was manual in 10 cases, all with transmesocolic ascending of the jejunal loop. There were $33 \%$ (n: 4) morbidity, all Clavien Dindo II, and 8\% mortality (n: 1). There was no leakage of the duodenojejunal anastomosis. During follow-up, none presented complications and 4 patients died of oncological progression. Conclusion: The main surgical indication was neoplastic pathology and in our series, there was no major morbidity or anastomotic leak.

Key Words: duodenal neoplasm; surgical procedures; distal duodenectomy.

\section{Resumen}

Introducción: La resección duodenal distal (RDD) es una técnica quirúrgica compleja e infrecuente usada para el tratamiento de patologías duodenales o extraduodenales, que no comprometen la papila duodenal mayor, siendo su indicación más frecuente la patología neoplásica. Objetivo: Dar a conocer nuestra experiencia con esta técnica quirúrgica. Materiales y Método: Serie de pacientes sometidos a RDD entre 2009-2020 en el Hospital Clínico UC. Resultados: Total 12 pacientes, mediana de edad 56 años. Diagnóstico preoperatorio: tumores primarios duodenales (4), tumores con compromiso duodenal por contigüidad (5) y patologías de urgencia (3). El abordaje fue abierto en 9 pacientes y laparoscópico en 3 ( 1 caso convertido). En 9 pacientes se resecó D3-D4, en 1 paciente incluyó parte de D2 y en 2, sólo D4. La anastomosis duodenoyeyunal fue manual en 10 casos, todas con ascenso transmesocólico del asa yeyunal. Hubo 33\% (n: 4) de morbilidad, todos Clavien Dindo II y $8 \%$ de mortalidad (n: 1). No hubo filtración de la anastomosis duodenoyeyunal. En el seguimiento ninguno presentó complicaciones y 4 pacientes fallecieron por progresión oncológica. Conclusión: La principal indicación quirúrgica fue patología neoplásica y en nuestra serie no hubo morbilidad mayor ni filtración anastomótica.

Palabras clave: neoplasias duodenales; procedimientos quirúrgicos; duodenectomía distal.

\section{Introducción}

El duodeno es un órgano que se caracteriza por sus complejas relaciones anatómicas, en especial con la desembocadura biliar y pancreática en la papila mayor y por su íntima cercanía con los vasos mesentéricos ${ }^{1}$. Su abordaje quirúrgico puede ser definido como proximal o distal, siendo el límite anatómico la papila duodenal mayor. En general, las lesiones con compromiso de la papila duodenal mayor requieren una resección en conjunto con la cabeza del páncreas y la vía biliar mediante una pancreatoduodenectomía, la cual se asocia a importante morbilidad y mortalidad. La patología que afecta al duodeno distal a la papila mayor se trata mediante una resección duodenal distal (RDD) la que puede ser total o segmentaria ${ }^{2}$.

Las RDD requieren un detallado conocimiento 
anatómico de la zona, siendo frecuente la necesidad de una adecuada exposición del retroperitoneo a través de las maniobras de Kocher y de Cattell-Bra$\mathrm{ash}^{3}$. Sus indicaciones son variadas, siendo lo más frecuente la patología neoplásica, principalmente GIST duodenal ${ }^{4}$. Con menor frecuencia se describen los adenocarcinomas duodenales, pero éstos habitualmente son de diagnóstico tardío, lo que limita su resecabilidad $^{5}$. Otros escenarios para RDD son los tumores primarios de órganos vecinos que invaden el duodeno por contigüidad, cirugía de urgencia por trauma y complicaciones vasculares como fístulas aorto-duodenales. La morbimortalidad está relacionada con la indicación de la cirugía y por la posibilidad del desarrollo de una fístula duodenal ${ }^{6}$.

La baja incidencia de tumores y patología quirúrgica de localización en duodeno distal hace que las RDD sean infrecuentes. Por lo mismo, la experiencia y documentación internacional de esta técnica es escasa. El objetivo de este artículo es dar a conocer la experiencia de nuestro centro en relación a esta técnica quirúrgica.

\section{Materiales y Método}

Este estudio corresponde a una serie de casos de pacientes sometidos a RDD en el Hospital Clínico de la Pontificia Universidad Católica de Chile entre los años 2009 y 2020. Se incluyó a todos los pacientes sometidos a resección D3 y/o D4, y D2 sin incluir la ampolla duodenal mayor. Los registros se obtuvieron desde la base de datos quirúrgica del centro y fueron tabulados y analizados con estadística descriptiva. Su publicación fue autorizada por el comité de ética de la institución.

\section{Resultados}

En total se realizaron 12 RDD en el período analizado. Los pacientes correspondieron a 6 hombres y 6 mujeres con una mediana de edad de 56 años. En cuanto al diagnóstico preoperatorio, 9 correspondían a etiología tumoral, 4 de ellos neoplasias primarias de duodeno: 3 GIST (Figura 1) y 1 adenocarcinoma, y 5 de localización extraduodenal con infiltración duodenal por contigüidad (3 por cáncer de colon) (Figuras 2 y 3). 8 pacientes requirieron una intervención adicional a la duodenectomía para resolver la patología de base (Tablas 1 y 2).

En cuanto a los detalles quirúrgicos, con respecto a la extensión de la duodenectomía, 9 pacientes requirieron resección de D3-D4, 2 pacientes sólo resección de D4 y 1 paciente requirió resección de D2 (inmediatamente distal a la papila duodenal mayor), D3 y D4. En cuanto al tipo de abordaje, 9 fueron cirugías abiertas en contexto de cirugía de urgencia y primario con invasión por contigüidad del duodeno y 3 laparoscópicas, 1 de estas últimas requirió conversión a vía abierta por la cercanía de la lesión a la papila. En cuanto a la técnica de la anastomosis, 10 fueron realizadas con sutura manual (Figura 4) y 2 con sutura mecánica. Con respecto al tipo de reconstrucción duodeno-yeyunal, 5 fueron termino-lateral, 6 latero-lateral y 1 termino-terminal.

En la serie hubo 5 pacientes que presentaron complicaciones (42\%), 4 ellos Clavien Dindo II (33\%), 3 pacientes requirieron apoyo con nutrición parenteral o enteral por sonda debido a íleo posoperatorio prolongado (2) y mal vaciamiento gástrico (1), 1 paciente requirió curaciones avanzadas con VAC debido a una infección de herida operatoria. Se observó 1 mortalidad (CD V 8\%), que corres-

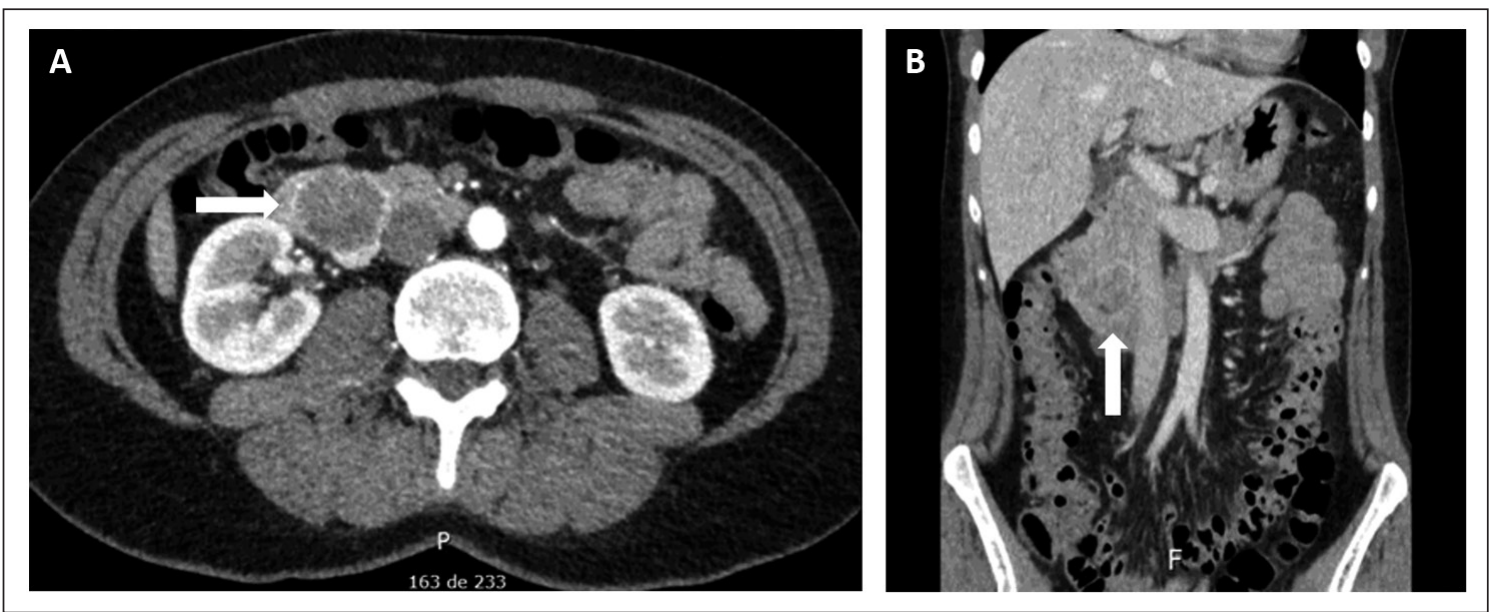

Figura 1. Caso 8. Tomografía que muestra GIST en la segunda porción del duodeno del (flechas blancas). A: Corte sagital. B: Corte coronal. 


\section{ARTÍ́CULO ORIGINAL}

Figura 2. Caso 10. Endoscopía digestiva alta de paciente con liposarcoma retroperitoneal con invasión de duodeno (flechas blancas), distal a la papila duodenal mayor (flecha negra).
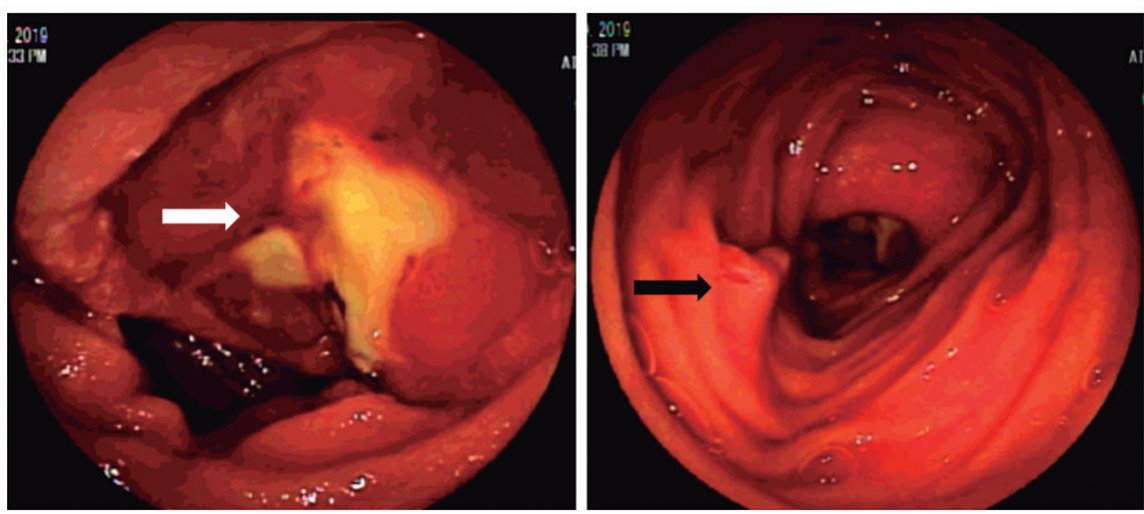

Figura 3. Caso 6. Tomografía de paciente con cáncer de colon derecho que infiltra duodeno distal (flechas blancas).
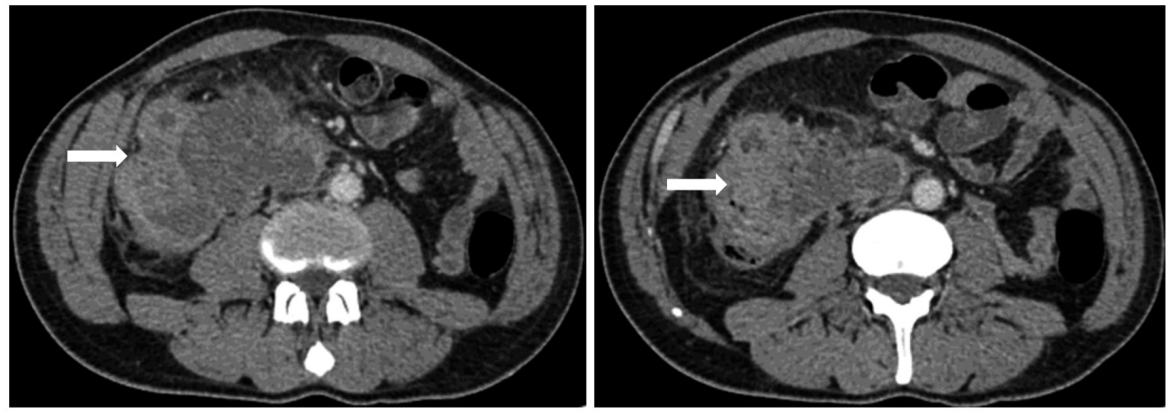

Tabla 1. Características de los pacientes (N: 12)

\begin{tabular}{|c|c|c|}
\hline Edad & $56(44)$ & md (rango) \\
\hline Género masculino & $6(50)$ & $\mathrm{N}(\%)$ \\
\hline Antecedentes mórbidos & $9(75)$ & $\mathrm{N}(\%)$ \\
\hline $\begin{array}{l}\text { ASA } \\
\text { I } \\
\text { II } \\
\text { III } \\
\text { IV }\end{array}$ & $\begin{array}{l}3(25) \\
4(33) \\
4(33) \\
1(8)\end{array}$ & $\begin{array}{l}\mathrm{N}(\%) \\
\mathrm{N}(\%) \\
\mathrm{N}(\%) \\
\mathrm{N}(\%)\end{array}$ \\
\hline $\begin{array}{l}\text { Diagnóstico preoperatorio } \\
\text { Neoplasias primarias de duodeno } \\
\text { GIST } \\
\text { Adenocarcinoma }\end{array}$ & $\begin{array}{l}4(33) \\
3(25) \\
1(8)\end{array}$ & $\begin{array}{l}\text { N }(\%) \\
\text { N }(\%) \\
\text { N }(\%)\end{array}$ \\
\hline $\begin{array}{l}\text { Otras neoplasias } \\
\text { Cáncer de colon con infiltración de duodeno } \\
\text { Tumor germinal } \\
\text { Liposarcoma retroperitoneal }\end{array}$ & $\begin{array}{c}3(25) \\
1(8) \\
1(8)\end{array}$ & $\begin{array}{l}\mathrm{N}(\%) \\
\mathrm{N}(\%) \\
\mathrm{N}(\%)\end{array}$ \\
\hline $\begin{array}{l}\text { Causas no tumorales } \\
\text { Perforación de víscera hueca } \\
\text { Fístula aorto-duodenal } \\
\text { Obstrucción intestinal }\end{array}$ & $\begin{array}{l}1(8) \\
1(8) \\
1(8)\end{array}$ & $\begin{array}{l}\text { N }(\%) \\
\text { N }(\%) \\
\text { N }(\%)\end{array}$ \\
\hline $\begin{array}{l}\text { Cirugía asociada } \\
\text { Hemicolectomía derecha extendida } \\
\text { Colecistectomía } \\
\text { Bypass protésico aorto-aórtico } \\
\text { Nefrectomía derecha }+ \text { hemicolectomía derecha }\end{array}$ & $\begin{array}{l}8(67) \\
3(25) \\
2(17) \\
1(8) \\
2(17)\end{array}$ & $\begin{array}{l}\text { N }(\%) \\
\text { N }(\%) \\
\text { N }(\%) \\
\text { N }(\%) \\
\text { N }(\%)\end{array}$ \\
\hline
\end{tabular}

ponde a un paciente con múltiples comorbilidades crónicas, con diagnóstico preoperatorio de cáncer de colon derecho con infiltración duodenal, el cual presentó una lesión parcial inadvertida de un conducto biliar segmentario posterior de implantación baja (Strasberg tipo C). Evolucionó con biliperitoneo que requirió reoperación precoz para aseo y drenaje con mejoría inicial, pero con descompensación cardiorrespiratoria posterior y falla renal secundaria, falleciendo finalmente a los 64 días desde la operación. Ningún paciente presentó filtración de la anastomosis duodenal (Tabla 3). La mediana del tiempo operatorio fue de $240 \mathrm{~min}$ y de la estadía hospitalaria fue de 11 días. El seguimiento promedio fue de 17 meses, donde ningún paciente presentó morbilidad alejada debido a la cirugía. Hasta la fecha, 4 pacientes han fallecido por progresión oncológica de la enfermedad de base.

\section{Discusión}

Esta serie está compuesta por 12 casos distribuidos en más de 10 años de experiencia, lo cual es concordante con la baja incidencia de etiologías que requieran este tipo de intervención. La etiología 
ARTÍCULO ORIGINAL

Tabla 2. Detalle quirúrgico por caso

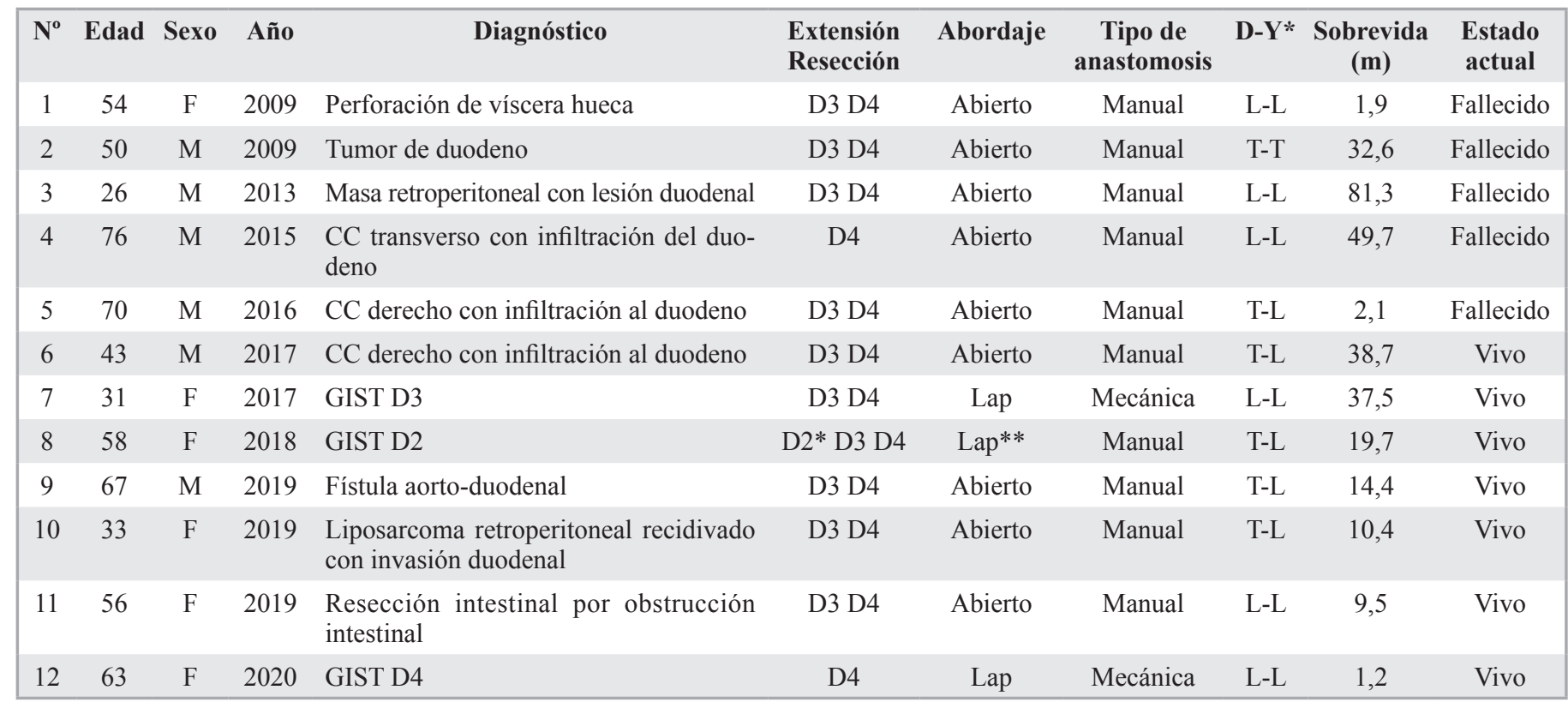

*Distal a la papila; **convertido.

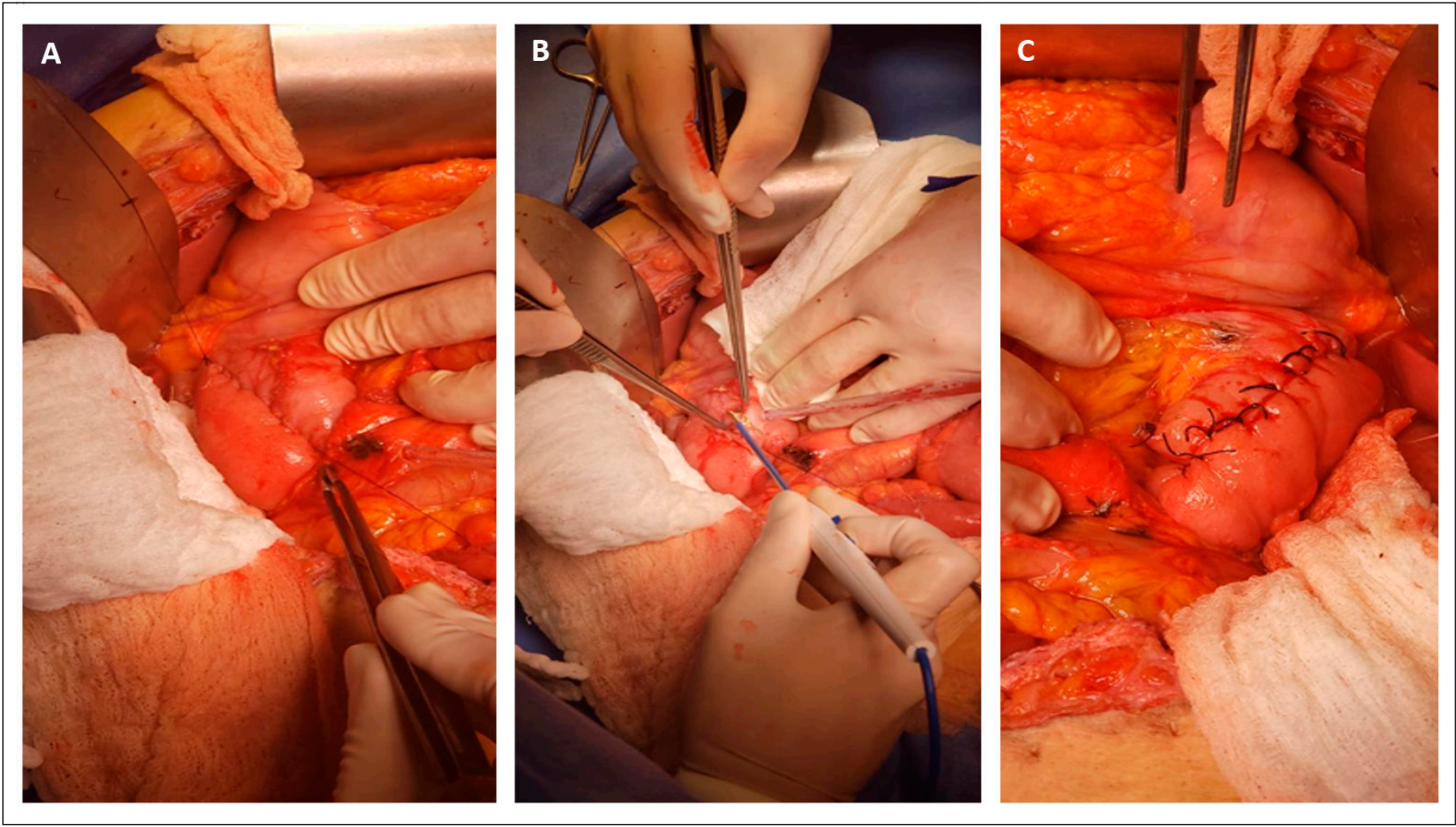

Figura 4. Caso 11. Anastomosis duodenoyeyunal latero-lateral manual. A: Afrontamiento de la pared posterior. B: Enterotomía. C: Sutura cara anterior (segundo plano). 
Tabla 3. Morbimortalidad y seguimiento

\begin{tabular}{|lrrc}
\hline Morbilidad general & 4 & $(33)$ & N (\%) \\
Clavien Dindo & & & \\
$\quad$ - II & 4 & $(33)$ & N (\%) \\
- V & 1 & $(8)$ & N (\%) \\
Morbilidad específica & & & \\
$\quad$ Fístula duodenal & 0 & $(0)$ & N (\%) \\
Mal vaciamiento gástrico & 1 & $(8)$ & N (\%) \\
Infección de herida operatoria & 1 & $(8)$ & N (\%) \\
Íleo prolongado & 2 & $(17)$ & N (\%) \\
Reoperaciones & 1 & $(8)$ & N (\%) \\
Tiempo operatorio (min) & $240(240)$ & md (rango) \\
Estadía hospitalaria (días) & 11 & $(59)$ & md (rango) \\
Seguimiento (meses) & 17 & $(80)$ & md (rango)
\end{tabular}

principal fue la patología neoplásica, siendo los tumores primarios duodenales un tercio de la serie, con sólo un caso de adenocarcinoma de duodeno distal y 5 pacientes con tumores que infiltraron el duodeno por contigüidad. La morbilidad fue baja, teniendo sólo un paciente con un cáncer avanzado que evolucionó con complicaciones graves que provocaron finalmente su fallecimiento. El abordaje más frecuente fue abierto y la anastomosis en la mayoría de los casos se realizó con sutura manual. Ningún paciente presentó filtración de la anastomosis duodenal.

La técnica quirúrgica del duodeno distal es desafiante por sus complejas relaciones anatómicas. El abordaje proximal o distal será definido por la necesidad o no de resecar la papila, para lo cual es fundamental la evaluación preoperatoria e incluso intraoperatoria de ésta mediante estudio endoscópico $^{6}$. Debe realizarse en centros de experiencia en pancreatoduodenectomías por el riesgo de compromiso de la papila, ya que estos pacientes presentarán una anatomía adversa para las anastomosis biliar y pancreática por tener ambos conductos habitualmente finos y páncreas de consistencia blanda. Las resecciones proximales revisten mayor morbilidad, alcanzando incluso en centros experimentados una morbilidad mayor de $35 \%-40 \%{ }^{7}$, y en patologías duodenales o extraduodenales puede ser mayor. Las RDD, cuando son posibles, ofrecen una alternativa bastante más segura, con una reconstrucción fisiológica del tracto digestivo que no expone al paciente a las complicaciones metabólicas de corto y largo plazo asociadas a la pancreatoduodenectomía. La indicación de RDD es infrecuente, y generalmente es debido a causa neoplásica, ya sea primaria de duodeno o infiltración por contigüidad. También en cirugía de urgencia por fístulas aorto-duodenales o trauma ${ }^{6}$. Las neoplasias duodenales primarias son raras y corresponden más frecuentemente a GIST. Por lo mismo, la evidencia se limita a series de casos que describen como único tratamiento curativo la resección ${ }^{8}$. Los adenocarcinomas de duodeno (excluyendo los ampulomas) son aún más raros, con un mal pronóstico debido a su diagnóstico tardío $0^{5,9,10}$. Es discutible si el mejor tratamiento de un adenocarcinoma duodenal distal a la papila corresponde a una pancreatoduodenectomía o a una RDD. El trabajo de Onkendi ${ }^{11}$, comparó ambas técnicas encontrando un mayor recuento ganglionar en pancreatoduodenectomía, pero sin diferencias en sobrevida a largo plazo. Por otro lado, los tumores de origen colorrectal han aumentado en incidencia y aquellos de localización derecha pueden comprometer el duodeno por vecindad, siendo necesario la resección en bloque con el colon derecho ${ }^{6}$. En el escenario de la cirugía de urgencia, las fístulas aorto-intestinales son de baja incidencia, pero en el $80 \%$ de los casos se producen por comunicación con el duodeno. Es una condición de riesgo vital que generalmente se manifiesta con shock hipovolémico ${ }^{12,13}$.

La cirugía del duodeno distal debe iniciarse con una adecuada exposición del retroperitoneo a través de una amplia maniobra de Kocher y de Cattell y Braash. Es necesario cerciorarse del margen con la papila mayor de tal manera de realizar una anastomosis proximal segura. En lesiones pequeñas y benignas, es posible realizar resecciones en cuña que obtengan un cierre adecuado a través de sutura primaria, o derivaciones con parche de yeyuno o en $\mathrm{Y}$ de Roux, sin obstruir el lumen ni la vascularización ${ }^{2}$. Para lesiones mayores y etiología maligna la resección debe tener una extensión mayor, desde D2, D3 o D4 a distal. El margen distal es preferentemente en el yeyuno, ya que posee vascularización desde la arcada de Rolando y, por lo tanto, tiene menor riesgo de filtraciones anastomóticas en comparación con la porción distal del duodeno de circulación terminal. Esta anastomosis puede ser realizada terminolateral, latero-lateral y termino-terminal, sin haber evidencia suficiente para apoyar una sobre la otra y en general es determinada por cada centro o caso a caso según las condiciones del paciente ${ }^{3}$.

Al igual que otras series expuestas en la literatura, nuestra serie está compuesta por pacientes electivos con patología neoplásica (Figuras 1, 2, 3) y pacientes de urgencia. Es destacable que, a pesar de la heterogenicidad de etiologías, la morbilidad general fue baja (33\% y sólo Clavien Dindo II) y 
debidas principalmente a mal vaciamiento gástrico e íleo posoperatorio. El caso de mortalidad estuvo marcado por una lesión infiltrativa en D2 en una paciente con múltiples comorbilidades en que la cirugía fue laboriosa y debido a las estrategias para conseguir el margen duodenal proximal se produjo una lesión biliar inadvertida. No tuvimos pérdidas en el seguimiento, y la sobrevida posterior a la cirugía estuvo marcada por el pronóstico de la patología subyacente, falleciendo 4 de los pacientes de causas no relacionadas con la cirugía. El abordaje fue abierto en la mayoría de los casos, dado que se requería una resección en bloque (colon, riñón) o correspondían a casos de urgencia. La técnica laparoscópica fue una buena alternativa para el manejo de GIST limitados al duodeno ${ }^{8}$.

Nuestra serie describe la experiencia con una técnica quirúrgica que, debido a lo infrecuente de su indicación, nos parece relevante dar a conocer.
Puede ser el escenario tanto en patología de urgencia como electiva, siendo fundamental tener las consideraciones técnicas mencionadas y considerarla una opción con menor morbilidad específica y mejor calidad de vida a largo plazo en relación con la pancreatoduodenectomía.

\section{Responsabilidades éticas}

Protección de personas y animales. Los autores declaran que para esta investigación no se han realizado experimentos en seres humanos ni en animales.

Confidencialidad de los datos. Los autores declaran que en este artículo no aparecen datos de pacientes.

Conflictos de interés: no hay.

\section{Bibliografía}

1. Blanc B, Valleur P. Résections duodénales et duodénojéjunales. EMC (Elsevier Masson SAS, Paris), Techniques chirurgicales - Appareil digestif. 2010;40415 .

2. Chung JC, Kim HC, Hur SM. Limited resections for duodenal gastrointestinal stromal tumors and their oncologic outcomes. Surg Today 2016;46:110-6.

3. Golhar A, Mangla V, Mehrotra S, Lalwani S, Mehta N, Nundy S. Limited distal duodenal resection: Surgical approach and outcomes. A case series. Ann Medicine Surg. 2018;30:36-41.

4. Shen Z, Chen P, Du N, Khadaroo PA, Mao D, Gu L. Pancreaticoduodenectomy versus limited resection for duodenal gastrointestinal stromal tumors: a systematic review and meta-analysis. BMC Surg. 2019;19:121. doi: 10.1186/s12893019-0587-4.

5. Stell D, Mayer D, Mirza D, Buckels J.
Delayed Diagnosis and Lower Resection Rate of Adenocarcinoma of the Distal Duodenum. Digest Surg. 2005;21:434-9.

6. Seman M, Kasereka-Kisenge F, Taieb A Noullet S, Trésallet $\mathrm{C}$. Resection of the third and four portions of the duodenum. J Visc Surg. 2018;156:45-9.

7. Giuliano K, Ejaz A, He J. Technical aspects of pancreaticoduodenectomy and their outcomes. Chin Clin Oncol. 2017;6:64-64.

8. Huang Y, Chen G, Lin L, Jin X, Kang $\mathrm{M}$, Zhang Y, et al. Resection of GIST in the duodenum and proximal jejunum: A retrospective analysis of outcomes. Eur J Surg Oncol. 2019;45:1950-6.

9. Lee CHA, Shingler G, Mowbray NG, Al $\square$ Sarireh B, Evans P, Smith M, et al. Surgical outcomes for duodenal adenoma and adenocarcinoma: a multicentre study in Australia and the United Kingdom. Anz J Surg. 2018;88:E157-E161.

10. Meijer L, Strijker M, Bakker J, Toennaer $\mathrm{J}$, Zonderhuis B, van der Vliet $\mathrm{H}$, et al. Clinical outcomes of patients with duodenal adenocarcinoma and intestinaltype papilla of Vater adenocarcinoma. World J Gastrointest Oncol. 2020;12:34757.

11. Onkendi EO, Boostrom SY, Sarr MG, Farnell MB, Nagorney DM, Donohue JH, et al. 15 Year Experience with Surgical Treatment of Duodenal Carcinoma: a Comparison of Periampullary and ExtraAmpullary Duodenal Carcinomas. J Gastrointest Surg. 2012;16:682-91.

12. Makimoto S, Takami T, Shintani H, Kataoka N, Yamaguchi T, Tomita M, et al. Cases of two patients with aortoduodenal fistula who underwent emergency operation. Int J Surg Case Reports 2020;69:87-91.

13. Ota K, Tatsumi Y, Matsui M, Yokoya Y, Tsuchimoto Y, Yano K, et al. Case of massive hematemesis by secondary aortic duodenal fistula 8 years after abdominal aortic aneurysm repair. Geriatr Gerontol Int. 2017;17:1234-5. 